\title{
Editorial: Physiological, Pathological Roles and Pharmacology of Insulin Regulated Aminopeptidase
}

\author{
Siew Yeen Chai ${ }^{1}$, Hugo Gutiérrez-de-Terán ${ }^{2}$ and Efstratios Stratikos ${ }^{3 *}$ \\ ${ }^{1}$ Department of Physiology, Monash Biomedicine Discovery Institute, Monash University, Clayton, VIC, Australia, \\ ${ }^{2}$ Department of Cell and Molecular Biology, Uppsala University, Uppsala, Sweden, ${ }^{3}$ Biochemistry Laboratory, Department of \\ Chemistry, National and Kapodistrian University of Athens, Athens, Greece
}

Keywords: aminopeptidase, metabolism, trafficking, immune system, peptide hormones, central nervous system, glucose, fibrosis

\section{Editorial on the Research Topic}

Physiological, Pathological Roles, and Pharmacology of Insulin Regulated Aminopeptidase

\section{OPEN ACCESS}

Edited and reviewed by: Cecilia Giulivi,

University of California, Davis, United States

*Correspondence: Efstratios Stratikos estratikos@chem.uoa.gr

Specialty section: This article was submitted to

Cellular Biochemistry,

a section of the journal

Frontiers in Molecular Biosciences

Received: 24 March 2021

Accepted: 30 March 2021

Published: 23 April 2021

Citation:

Chai SY, Gutiérrez-de-Terán H and Stratikos E (2021) Editorial: Physiological, Pathological Roles and Pharmacology of Insulin Regulated Aminopeptidase.

Front. Mol. Biosci. 8:685101. doi: 10.3389/fmolb.2021.685101
Insulin-Regulated Aminopeptidase (IRAP) is a transmembrane zinc metalloprotease with several reported biological functions. IRAP belongs to the M1 family of aminopeptidases (EC 3.4.11.3) and is also known as cystinyl aminopeptidase, placental leucine aminopeptidase (PLAP), and oxytocinase. The reported biological functions of IRAP include: (i) the regulation of trafficking of glucose transporter 4 (Keller, 2003), (ii) the generation of antigenic peptides for cross-presentation (Saveanu et al., 2009), (iii) T-cell receptor signaling (Evnouchidou et al., 2020), (iv) the regulation of placental oxytocin levels (Tsujimoto et al., 1992), (v) not well-understood roles in cognition and other central nervous system functions possibly through the regulation of oxytocin and vasopressin levels in the brain (Herbst et al., 1997; Albiston et al., 2011; Bernstein et al., 2017) or altered glucose uptake (Fernando et al., 2008; Albiston et al., 2011; Ismail et al., 2017), and (vi) the regulation of organ fibrosis (T. Gaspari, personal communication). All these roles have been associated with at least one of the two functional components of IRAP: an extracellular $C$-terminal domain that contains the M1 exopeptidase catalytic site and a 110 amino-acid long cytosolic $\mathrm{N}$-terminal domain, connected by a single transmembrane-spanning region. The $C$-terminal domain underlies the ability of IRAP to trim antigenic peptides and peptide hormones, whereas the $\mathrm{N}$-terminal domain appears to control intracellular trafficking and signaling events. The structure of the extracellular domain has been recently solved and resembles several other enzymes of the M1 family of aminopeptidases, featuring a large internal cavity adjacent to the catalytic center, which can accommodate peptide substrates (Mpakali et al., 2015) (Figure 1). The C-terminal domain can dimerize and change conformations upon ligand binding (Mpakali et al., 2017). Very little is currently known about the structure and molecular interactions of the $\mathrm{N}$-terminal domain.

The important biological functions in which IRAP participates are attracting increased attention for possible pharmacological interventions. The primary function targeted to date has been the aminopeptidase activity, for which both functional and structural knowledge exists. In particular, IRAP inhibitors have been pursued as potential therapeutics for cognitive disorders (Chai et al., 2008; Andersson and Hallberg, 2012; Diwakarla et al., 2016), immune modulators (Kokkala et al., 2016), and more recently as anti-fibrotic agents.

In this special issue "Physiological, Pathological Roles and Pharmacology of Insulin Regulated Aminopeptidase," we present a series of reviews and research papers written from leading authors in the field of IRAP, covering most aspects of the state-of-the-art research for this enzyme. 


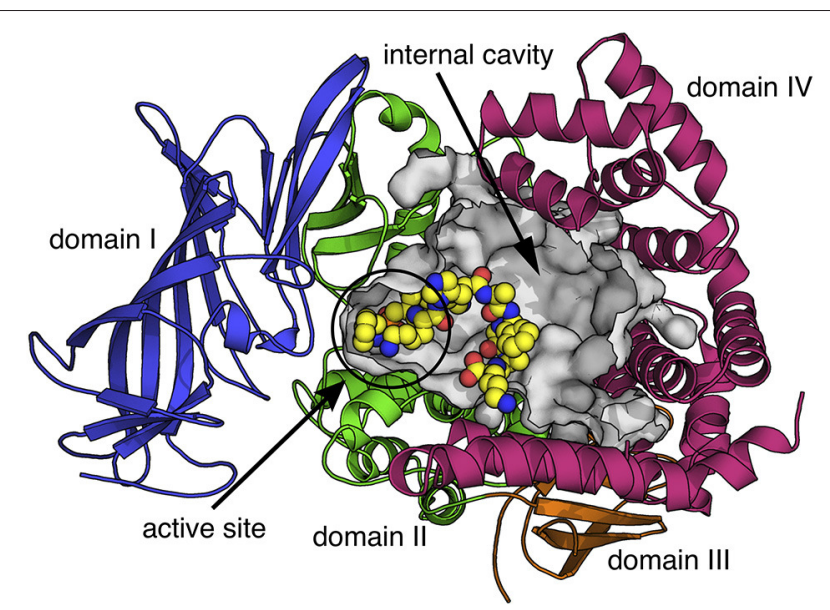

FIGURE 1 | Schematic representation of the extracellular domain of IRAP based on the crystal structure with PDB code 4Z7I (Mpakali et al., 2015). Protein is depicted in cartoon representation with the four domains indicated in different colors. The internal cavity that contains the aminopeptidase active site and can accommodate peptide substrates is shown as a gray surface. A peptide analog co-crystallized with IRAP is shown in sphere representation (carbon $=$ yellow, nitrogen $=$ blue, oxygen $=$ red, phosphorus $=$ orange) .

With regards to the role of IRAP in metabolism, Trocmé et al. explore the possibility of using serum IRAP as a novel biomarker of prediabetes and type 2 diabetes, Krskova et al. demonstrate that IRAP inhibition improves glucose clearance in obese Zucker rats and Segarra et al. demonstrate how different types of dietary fat intake can affect IRAP and Alanyl aminopeptidase activities in the frontal cortex of the brain, the liver, and plasma. Exploring the role of IRAP in the immune system, Weimershaus et al. demonstrate how IRAP endosomes control phagosomal maturation in dendritic cells and Descamps et al. explore the role of IRAP in endocytic trafficking and receptor signaling in immune cells. Related to the role of IRAP in the central nervous system, Goto et al. describe a reciprocal relationship between IRAP expression and vasopressin levels in the murine brain.

\section{REFERENCES}

Albiston, A. L., Diwakarla, S., Fernando, R. N., Mountford, S. J., Yeatman, H. R., Morgan, B., et al. (2011). Identification and development of specific inhibitors for insulin-regulated aminopeptidase as a new class of cognitive enhancers. $\mathrm{Br}$. J. Pharmacol. 164, 37-47. doi: 10.1111/j.1476-5381.2011.01402.x

Andersson, H., and Hallberg, M. (2012). Discovery of inhibitors of insulinregulated aminopeptidase as cognitive enhancers. Int. J. Hypertens. 2012:789671. doi: 10.1155/2012/789671

Bernstein, H. G., Müller, S., Dobrowolny, H., Wolke, C., Lendeckel, U., Bukowska, A., et al. (2017). Insulin-regulated aminopeptidase immunoreactivity is abundantly present in human hypothalamus and posterior pituitary gland, with reduced expression in paraventricular and suprachiasmatic neurons in chronic schizophrenia. Eur. Arch. Psychiatry Clin. Neurosci. 267, 427-443. doi: 10.1007/s00406-016-0 757-7

Chai, S. Y., Yeatman, H. R., Parker, M. W., Ascher, D. B., Thompson, P. E., Mulvey, H. T., et al. (2008). Development of cognitive enhancers based on
In view of the two functional domains of IRAP, Vear et al. explore the under-studied relationship between the $N$-terminal cytosolic and the C-terminal catalytic domains of IRAP and how the localization of IRAP may play an important role in defining its physiological or pathological functions. Finally, a number of articles explore the development of IRAP inhibitors with potential pharmacological and chemical biology applications: Georgiadis et al. provide a broad review on the development of IRAP inhibitors, Barlow and Thompson focus on efforts to develop inhibitors inspired by other members of the M1 family of aminopeptidases, Hallberg et al. review the development of angiotensin IV inspired small peptidemimetic inhibitors for IRAP and Vanga et al. explore the structural basis of inhibition of IRAP by benzopyran-based compounds.

It is becoming clear that the important biological functions played by IRAP will undoubtably sustain scientific interest on this enzyme in the coming years, especially in view of newly discovered functions with poorly understood molecular mechanisms. However, its multitude of biological roles could also act as a deterrent for drug development efforts. Likely, IRAPs tractability as a pharmaceutical target will greatly depend on detailed mechanistical analysis and prioritization of its biological functions, an aspect in which this Special Issue contributes. On the other hand, solid pre-existing progress in inhibitor design and development could incentivize drug development efforts as highlighted by contributed articles to this Special Issue. The possible effect of active-site inhibitors to IRAP's trafficking functions needs to be carefully addressed, an aspect that will certainly benefit from further work on the interplay of the two domains of IRAP. Regardless of these potential caveats, one thing is clear: the coming together of IRAP researchers from different fields and the continued frank and honest discussion that ensues will ensure that progress will be made, paving the way for the validation of IRAP as a tractable therapeutic target in the future.

\section{AUTHOR CONTRIBUTIONS}

All authors contributed to the writing of the editorial and have approved the final version. inhibition of insulin-regulated aminopeptidase. BMC Neurosci. 9(Suppl. 2):S14. doi: 10.1186/1471-2202-9-S2-S14

Diwakarla, S., Nylander, E., Gronbladh, A., Vanga, S. R., Khan, Y. S., Gutierrezde-Teran, H., et al. (2016). Binding to and inhibition of insulin-regulated aminopeptidase by macrocyclic disulfides enhances spine density. Mol. Pharmacol. 89, 413-424. doi: 10.1124/mol.115.102533

Evnouchidou, I., Chappert, P., Benadda, S., Zucchetti, A., Weimershaus, M., Bens, M., et al. (2020). IRAP-dependent endosomal T cell receptor signalling is essential for $\mathrm{T}$ cell responses. Nat. Commun. 11:2779. doi: 10.1038/s41467-020-16471-7

Fernando, R. N., Albiston, A. L., and Chai, S. Y. (2008). The insulin-regulated aminopeptidase IRAP is colocalised with GLUT4 in the mouse hippocampuspotential role in modulation of glucose uptake in neurones? Eur. J. Neurosci. 28, 588-598. doi: 10.1111/j.1460-9568.2008.06347.x

Herbst, J. J., Ross, S. A., Scott, H. M., Bobin, S. A., Morris, N. J., Lienhard, G. E., et al. (1997). Insulin stimulates cell surface aminopeptidase activity toward vasopressin in adipocytes. Am. J. Physiol. 272, E600-E606. doi: 10.1152/ajpendo.1997.272.4.E600 
Ismail, M. A. M., Mateos, L., Maioli, S., Merino-Serrais, P., Ali, Z., Lodeiro, M., et al. (2017). 27-Hydroxycholesterol impairs neuronal glucose uptake through an IRAP/GLUT4 system dysregulation. J. Exp. Med. 214, 699-717. doi: 10.1084 /jem. 20160534

Keller, S. R. (2003). The insulin-regulated aminopeptidase: a companion and regulator of GLUT4. Front. Biosci. 8, s410-s420. doi: 10.2741/1078

Kokkala, P., Mpakali, A., Mauvais, F.-X., Papakyriakou, A., Daskalaki, I., Petropoulou, I., et al. (2016). Optimization and structure-activity relationships of phosphinic pseudotripeptide inhibitors of aminopeptidases that generate antigenic peptides. J. Med. Chem. 59, 9107-9123. doi: 10.1021/acs.jmedchem.6b01031

Mpakali, A., Saridakis, E., Harlos, K., Zhao, Y., Kokkala, P., Georgiadis, D., et al. (2017). Ligand-induced conformational change of insulin-regulated aminopeptidase: insights on catalytic mechanism and active site plasticity. J. Med. Chem. 60:2963-2972. doi: 10.2210/pdb5mj6/pdb

Mpakali, A., Saridakis, E., Harlos, K., Zhao, Y., Papakyriakou, A., Kokkala, P., et al. (2015). Crystal structure of insulin-regulated aminopeptidase with bound substrate analogue provides insight on antigenic epitope precursor recognition and processing. J. Immunol. 195, 2842-2851. doi: 10.4049/jimmunol.1501103
Saveanu, L., Carroll, O., Weimershaus, M., Guermonprez, P., Firat, E., Lindo, V., et al. (2009). IRAP identifies an endosomal compartment required for MHC Class I cross-presentation. Science 325, 213-217. doi: 10.1126/science.1172845 Tsujimoto, M., Mizutani, S., Adachi, H., Kimura, M., Nakazato, H., and Tomoda, Y. (1992). Identification of human placental leucine aminopeptidase as oxytocinase. Arch. Biochem. Biophys. 292, 388-392. doi: 10.1016/0003-9861(92)90007-J

Conflict of Interest: The authors declare that the research was conducted in the absence of any commercial or financial relationships that could be construed as a potential conflict of interest.

Copyright (C) 2021 Chai, Gutiérrez-de-Terán and Stratikos. This is an open-access article distributed under the terms of the Creative Commons Attribution License (CC $B Y)$. The use, distribution or reproduction in other forums is permitted, provided the original author(s) and the copyright owner(s) are credited and that the original publication in this journal is cited, in accordance with accepted academic practice. No use, distribution or reproduction is permitted which does not comply with these terms. 